\title{
Lower Trapezius Muscle Transfer For Elbow Extension Reconstruction After Failed Nerve Transfer for Tetraplegia
}

\author{
Jayme Bertelli* and Sushil Nehete \\ Department of Neurosurgery, Brazil
}

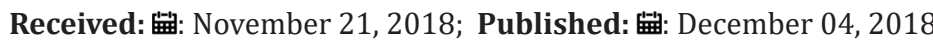

*Corresponding author: Jayme Bertelli, Hospital Celso Ramos, Department of Neurosurgery, Brazil

\begin{abstract}
Reconstruction of elbow extension is a first priority in the management of tetraplegia patients. Traditionally, posterior deltoid and biceps to triceps muscle transfers were used; but, in recent years, nerve transfers have become the preferred choice of a few surgeons. Nerve transfer reconstruction failures exist, however, often related to poor donor nerves, prolonged intervals between the injury and surgery and advanced patient age. As a valid surgical alternative in such cases, we propose transferring the lower trapezius muscle to reconstruct elbow extension, since this procedure already is being performed successfully in brachial plexus injury patients. We now report two patients in whom a lower trapezius transfer was employed as a successful salvage procedure after failed nerve-transfer surgery.
\end{abstract}

Keywords: Brachial Plexus; Tetraplegia; Nerve Transfer; Trapezio Muscle; Elbow Extension

\section{Introduction}

For the last several years, it has been emphasized that restoring upper limb function takes precedence over any other objective during the rehabilitation of traumatic tetraplegia patients [1] According to an IFSSH (International Federation of Societies for Surgery of the Hand) scientific committee update [2] on hand surgery in tetraplegic patients, one of the main goals of reconstruction is elbow extension [2] Traditionally, elbow extension has been reconstructed by transferring the posterior deltoid [3] or biceps muscle [4]. Recently, transferring the posterior division of the axillary nerve or the motor branch to the brachialis to triceps branches has demonstrated consistent recovery of M4 elbow extension strength; thereby supplanting muscle transfers [5]. Failed nerve transfer reconstructions exist, however, often related to poor donor nerves, a prolonged interval between the injury and surgery, and more advanced patient age. In this situation, salvage surgery is complicated. On one hand, if surgeons have used the posterior division of the axillary nerve as the donor, the posterior deltoid muscle is unavailable for transfer. The biceps muscle would still be available.

However, concomitant to triceps reconstruction, thumb and finger extension are typically reconstructed by transferring the nerve to supinator to the PIN [5] In the case of a biceps transfer, supination will be lost. In our previous cases, when we used the biceps to triceps muscle transfer, some of our patients complained about lost elbow flexion strength. They particularly noticed this when they hooked their flexed arm around their thigh to change lower limb position (unpublished observations), a complication that others have also recently reported [6]. In fact, a roughly $50 \%$ decrease in elbow flexion strength is routine after a biceps- to triceps transfer [7] which makes us reluctant to propose this surgery in the first place. Bertelli et al. [8] has successfully transferred the lower trapezius muscle to reconstruct elbow extension in patients with brachial plexus injuries. We hypothesised that this technique could [3] also be useful to reconstruct elbow extension among tetraplegics when a nerve transfer fails to achieve adequate results. In this paper, we present two patients with traumatic tetraplegia who had improved inadequately after a nerve transfer procedure and subsequently underwent a lower trapezius transfer to reconstruct elbow extension.

\section{Case Reports}

In advance of any data collection, the protocol of the present study was approved by our local ethics committee. Each patient provided his verbal informed consent before participating, in accordance with the Declaration of Helsinki guiding biomedical 
research involving human subjects (http://www.cirp.org/library/ ethics/helsinki/).

\section{Case 1}

A17-year-old gentleman presented with tetraplegiafollowing an injury that he sustained diving into shallow water. On examination, nine months after the accident, he was found to be Group 1 on the right and Group 2 on the left, as per the International Classification (IC) for Surgery of the Hand in Tetraplegia. He had bilateral elbow flexion contractures. We planned nerve transfer surgeries for elbow extension, along with other nerve transfers, to enhance function in both of his hands. Bilaterally, the posterior division of the axillary nerve was transferred to the nerve innervating the long and upper medial heads of the triceps via axillary access, as reported elsewhere [5]. At 18-month follow up, elbow extension scored M4 on the right side, as rated using the British Medical Research Council (BMRC) scale grading muscle strength. However, left elbow extension strength only scored M2, which had not improved even when he was reassessed at 24 months post operatively. At that time, we performed a lower trapezius transfer to the triceps to reconstruct left elbow extension. Sixteen months after this second surgery, elbow extension scored M4 in the horizontal position. The patient was able to extend his elbow with his shoulder at $1000 \mathrm{C}$ of abduction, but 4 not in full abduction. However, he was able to keep his elbow extended with his shoulder fully abducted, which had been impossible for him prior to the trapezius transfer. Video 1 shows pre- and postoperative results for Case 1 post lower trapezius transfer.

\section{Case 2}

A 21-year-old man presented one year following a road traffic accident in which he sustained a spinal cord injury and was left tetraplegic. He scored as IC Group 2 for tetraplegia on the right and Group 3 on the left. Wrist extension scored M2 and M4 on the right and left, respectively. He exhibited no recovery of right-sided triceps function even 36 months after a posterior division of the axillary nerve transfer to the triceps long head motor branch. By then, left triceps strength had improved to M4. At this time, a trapezius-totriceps transfer was performed to restore right elbow extension. Twenty-four months after this surgery, the patient was able to extend his elbow with his shoulder at 90 degrees of abduction, scoring M3. Video 2 documents the pre- and postoperative views after lower trapezius transfer in Case 2.

\section{Surgical Technique}

Under general Anaesthesia without relaxants, each patient was placed in a lateral decubitus position. An incision was made from the spine of the scapula to the T12 vertebra. The trapezius muscle was harvested from the T12 vertebra, in continuity with a $1 \mathrm{~cm}$ wide strip of fascia over the spinous processes of T12 to L3. The trapezius was detached from the spine up to C7. Care was taken not to injure the rhomboid muscles (Figure 1). The XI spinal accessory nerve was identified and protected. The medial side of the lower trapezius, which was inserted into the spine, now was sutured to the medial border and spine of the scapula. A $10 \mathrm{~cm}$-long incision was created over the posterior surface of the shoulder and upper arm, and 5 another one, again $10 \mathrm{~cm}$ long, over the triceps tendon. These incisions were made to permit tunnelling of the lower trapezius to the triceps tendon. With the arm adducted, shoulder extended, and elbow fully extended, the lower trapezius was sutured to the triceps tendon under maximal tension. In Case 2, a portion of lumbar fascia was removed to reinforce the lower trapezius tendon (Figure 2). After skin closure, the upper extremity was immobilised in a plaster cast, with the arm completely adducted and extended 30o, and the elbow flexed 20o. The shoulder was placed in $30 \mathrm{o}$ of extension to allow for some relaxation of the transplanted muscle. The cast was maintained for four weeks. Over this period, patients were asked to individualise their lower trapezius and contract it 10 times per cycle, three cycles per day. To instruct patients how to individualise lower trapezius muscle function, the surgeon gently squeezed the transferred muscle and asked the patient to contract where they felt pressure.
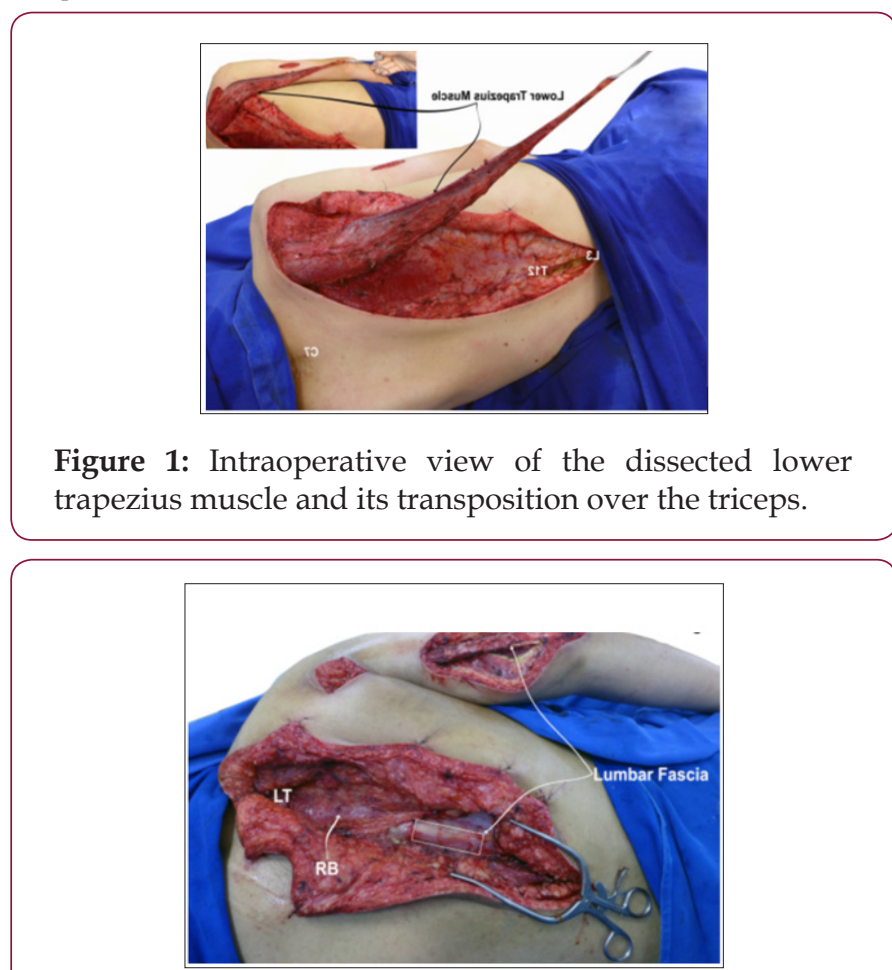

Figure 2: Intraoperative view of the dissected lower trapezius muscle and its transposition over the triceps. A segment of lumbar fascia was harvested to reinforce the lower trapezius muscle's attachment to the triceps tendon. (LT) lower trapezius sutured to the medial margin of the scapula, (RB) rhomboid muscles.

\section{Discussion}

Even though not strong enough to permit patients to raise their body up for transfer, the restoration of elbow extension was useful for improving arm positioning and, thereby, increasing its functional range. Before attempting this surgical technique, we were concerned that removing the lower trapezius might impair trunk control. This did not happen. In fact, both patients reported better trunk control after surgery: while sitting, they now used their restored elbow extension to control their balance, using the 
arm rest of the wheel-chair as support. We propose using the lower trapezius as an alternative for triceps reanimation for various reasons. First, trapezius transfers have already been employed successfully to reconstruct elbow extension in patients with brachial plexus injuries [8,9]. Second, the trapezius is innervated by the spinal accessory nerve, which has a cranial origin in the medulla and a spinal origin from the upper five cervical roots. Since both these components should be uninjured in surviving tetraplegics, the availability of the trapezius for muscle transfer should never be an issue.

Third, postoperative re-education was straight forward: squeezing the transferred belly of the trapezius muscle and asking patients to contract where they felt pressure helped them learn how to selectively contract their lower trapezius. Holtermann et al. [10] observed that all his volunteers were capable of independent control of the lower trapezius when examined by EMG. They believed that this resulted from the selective contribution of the cervical plexus to lower trapezius innerve [10]. The lower trapezius muscle also can be used, not only as a fall-back option, as in our patients, but in patients in whom the deltoid and biceps are unavailable for transfer. A final advantage of the proposed surgery is that the lower trapezius muscle's insertion can be extended by harvesting fascia from the T12 to L3 spinal processes, thereby increasing the tendon's length and eliminating any need for a tendon graft. In tetraplegic patients, our first choice for reconstructing elbow extension is to perform a nerve transfer. However, whenever transferring a nerve fails, transferring the lower trapezius muscle to the triceps is a safe and effective alternative.

ISSN: 2574-1241

DOI: 10.26717/BJSTR.2018.11.002139

Jayme Bertelli. Biomed J Sci \& Tech Res (C) (P) This work is licensed under Creative

Submission Link: https://biomedres.us/submit-manuscript.php

\section{References}

1. Snoek GJ, Ijzerman MJ, Hermens HJ, Maxwell D, Biering Sorensen F (2004) Survey of the needs of patients with spinal cord injury: Impact and priority for improvement in hand function in tetraplegics. Spinal Cord 42: 526-532.

2. Clarke H, Tse R, Malessy M, Kozin S (2015) IFSSH Scientific Committee on Neonatal Brachial Plexus Palsy The Role of Nerve Transfers in the Treatment of Neonatal Brachial Plexus Palsy. IFSSH Ezine 5(2): 25-38.

3. Bryden A, Peljovich A, Hoyen H, Nemunaitis G, Kilgore K, et al. (2012) Surgical Restoration of Arm and Hand Function in People with Tetraplegia. Top Spinal Cord Inj Rehabil 18(1): 43-49.

4. Kuz JE, Van Heest AE, House JH (1999) Biceps-to-triceps transfer in tetraplegic patients: Report of the medial routing technique and followup of three cases. J Hand Surg Am 24(1): 161-172.

5. Bertelli JA, Ghizoni MF (2015) Nerve transfers for elbow and finger extension reconstruction in midcervical spinal cord injuries. J Neurosurg 122(1): 121-127.

6. Medina J, Marcos García A, Jiménez I, Muratore G, Méndez Suárez JL (2017) Biceps to Triceps Transfer in Tetraplegic Patients: Our Experience and Review of the Literature. Hand 12(1): 85-90.

7. Mulcahey MJ, Lutz C, Kozin SH, Betz RR (2003) Prospective Evaluation of Biceps to Triceps and Deltoid to Triceps for Elbow Extension in Tetraplegia. J Hand Surg Am 13(2): 964-971.

8. Bertelli JA (2009) Lower trapezius muscle transfer for reconstruction of elbow extension in brachial plexus injuries. J Hand Surg Eur Vol 34(4): 459-464.

9. Alrabai HM, Gesheff MG, Hammouda AI, Conway JD (2018) Trapezius Muscle Transfer for Restoration of Elbow Extension in a Traumatic Brachial Plexus Injury. J Hand Surg 43(9): 872.

10. Holtermann A, Roeleveld K, Mork PJ, Grönlund C, Karlsson JS, et al. (2009) Selective activation of neuromuscular compartments within the human trapezius muscle. J Electromyogr Kinesiol 19(5): 896-902.

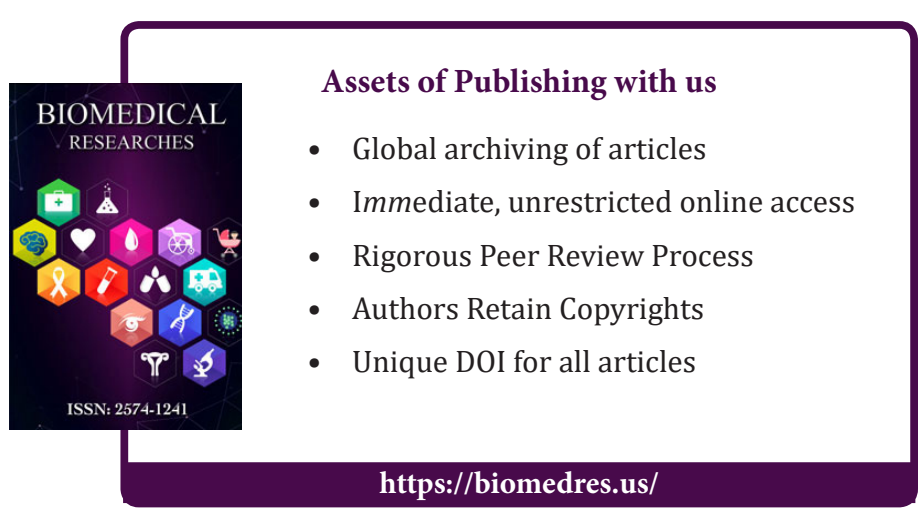

